\title{
Accurate Isolation Networks in Quadrature Couplers and Power Dividers
}

\author{
Batuhan Sutbas ${ }^{\circledR}$, Ekmel Ozbay ${ }^{\circledR}$, Member, IEEE, and Abdullah Atalar ${ }^{\circledR}$, Fellow, IEEE
}

\begin{abstract}
When quadrature couplers and power dividers are implemented in integrated circuits, accurate isolation networks can not be realized due to the nonideal resistors and the process variations. We present an isolation network design technique which cancels the resistor parasitic effects and also increases the tolerance to variations in the resistance values. A Lange coupler and a power divider are designed at $K a$-band using the proposed accurate and process-tolerant isolation networks. The improvement is analytically shown and empirically verified with our in-house GaN-based microstrip MMIC process. For the coupler, the measured return losses and isolation are better than $20 \mathrm{~dB}$ from DC to $40 \mathrm{GHz}$. The power divider achieves $20 \mathrm{~dB}$ return losses and isolation in a fractional bandwidth of $50 \%$. Both devices maintain $20 \mathrm{~dB}$ performance even when the variation in sheet resistance is as high as $\mathbf{3 0 \%}$.
\end{abstract}

Index Terms-Accurate, parasitic effect, wideband, quadrature coupler, power divider, isolation, sheet resistance, processtemperature variation, tolerance, integrated circuit.

\section{INTRODUCTION}

Q UADRATURE couplers and power dividers are widely used for splitting and combining RF power at the input and output of balanced amplifiers [1]. Such configurations require the isolation ports of the quadrature couplers to be terminated with $50 \Omega$ resistors. Also, power dividers use resistors in their isolation networks.

Overall circuit performance deteriorates when return loss and isolation parameters of the splitting structure degrades. Therefore, isolation networks of the couplers and dividers must be accurate and robust to variations in process and temperature. The accuracy is vital especially for millimeter-wave integrated circuits since the resistor parasitic effects become

Manuscript received August 5, 2020; revised September 15, 2020 and October 10, 2020; accepted November 1, 2020. Date of publication November 3, 2020; date of current version March 26, 2021. This brief was recommended by Associate Editor Y. Qin. (Corresponding author: Batuhan Sutbas.)

Batuhan Sutbas was with the Department of Electrical and Electronics Engineering, Bilkent University, 06800 Ankara, Turkey, and also with the Nanotechnology Research Center, Bilkent University, 06800 Ankara, Turkey. $\mathrm{He}$ is now with the Circuit Design Department, Innovations for High Performance Microelectronics, 15236 Frankfurt (Oder), Germany (e-mail: sutbas@ee.bilkent.edu.tr).

Ekmel Ozbay is with the Nanotechnology Research Center, Bilkent University, 06800 Ankara, Turkey, and also with the Department of Electrical and Electronics Engineering, Bilkent University, 06800 Ankara, Turkey (e-mail: ekmel@ee.bilkent.edu.tr).

Abdullah Atalar is with the Department of Electrical and Electronics Engineering, Bilkent University, 06800 Ankara, Turkey (e-mail: atalar@ee.bilkent.edu.tr).

Color versions of one or more figures in this article are available at https://doi.org/10.1109/TCSII.2020.3035667.

Digital Object Identifier 10.1109/TCSII.2020.3035667 dominant at high frequencies and post-fabrication tuning is quite difficult [2]. In order to reduce the resistor parasitic effects, isolation networks without direct ground connections are used [3] and compensation elements are also added for better matching [4]. However, such techniques are inadequate, as they are tied to accurate resistor models and do not provide tolerance to variations in the resistance values. Optimizing the fabrication process [5] is always helpful, yet it is a costly investment and perfection is never reached, therefore, an effort on the design part is unavoidable. Use of resistors can be completely abandoned [6], but the resulting bandwidth is narrow. In this brief, we analyze the effect of nonideal resistors in couplers and dividers, and propose accurate isolation networks which cancel the resistor parasitic effects and are also tolerant to systematic errors in the resistance values.

More recently, frequency tunable quadrature couplers [7] and power dividers [8] are presented at relatively low frequencies where the resistor parasitic effects are negligible. Our proposed isolation networks can also be used in such circuits to mitigate the performance degradation at higher frequencies as well as providing tolerance to sheet resistance variance.

\section{ANALYSIS AND DESIGN}

\section{A. Nonideal Resistors in Integrated Circuits}

Lumped element resistors are used at the isolation port of quadrature couplers and at the isolation network of power dividers. These resistors are typically realized by thinfilm technology in monolithic microwave integrated circuits (MMIC). Sheet resistance of the film is a function of the resistive material and its thickness. However, due to the reproducibility limitations in the manufacturing process, the sheet resistance is not a constant and the designer needs to account for certain variances. In our analysis, we use $\rho$ to denote the normalized sheet resistance which is ideally equal to 1 .

A simple high frequency model for a thin-film resistor is shown in Fig. 1. Here, $L_{i}$ is the inductance due to the finite length of the resistor and $C_{i}$ is the capacitance due to the finite distance from the resistor to the dielectric substrate. Both parameters are a function of the resistor geometry. The intrinsic resistance $R_{i}$ is scaled by $\rho$, and we assume resistors in close proximity are scaled by the same factor, since they undergo the same manufacturing process. 


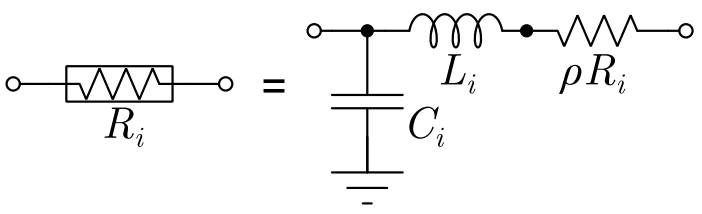

Fig. 1. Simple high frequency model for a thin-film resistor.

\section{B. Termination Accuracy in Quadrature Couplers}

Frequency response of a four-port quadrature coupler can be represented by an S-parameter matrix $S=\left[S_{i j}\right]$, where $i, j \in$ $\{1,2,3,4\}$. We consider the first port as the input port, the third port as the output port with $90^{\circ}$ phase difference, and the fourth port as the isolation port. The symmetrical S-parameter matrix of a quadrature coupler is given as

$$
[S]=\left[\begin{array}{cccc}
\Gamma_{0} & F_{c} & F_{d} & I_{0} \\
F_{c} & \Gamma_{0} & I_{0} & F_{d} \\
F_{d} & I_{0} & \Gamma_{0} & F_{c} \\
I_{0} & F_{d} & F_{c} & \Gamma_{0}
\end{array}\right]
$$

When the isolation port of a quadrature coupler is terminated with an imperfect load with a reflection coefficient of $\Gamma_{L}$, the S-parameters of the resulting three-port network $\bar{S}=\left[\bar{S}_{i j}\right]$ are found using Mason's rule [9] approximately as

$$
\left|\bar{S}_{i j}\right|=\left|S_{i j}+\frac{S_{i 4} S_{4 j} \Gamma_{L}}{1-\Gamma_{0} \Gamma_{L}}\right| \approx\left|S_{i j}+S_{i 4} S_{4 j} \Gamma_{L}\right|
$$

when the reflection coefficient of the coupler and the reflection at the isolation port are small with $\left|\Gamma_{0} \Gamma_{L}\right| \ll 1$.

Input reflection and transmission coefficients of the threeport network are barely affected by the accuracy of the coupler termination, as $\left|I_{0} \Gamma_{L}\right| \ll 1$.

$$
\begin{aligned}
& \left|\bar{S}_{11}\right| \approx\left|\Gamma_{0}+I_{0}^{2} \Gamma_{L}\right| \approx\left|S_{11}\right| \\
& \left|\bar{S}_{21}\right| \approx\left|F_{c}+F_{d} I_{0} \Gamma_{L}\right| \approx\left|S_{21}\right| \\
& \left|\bar{S}_{31}\right| \approx\left|F_{d}+F_{c} I_{0} \Gamma_{L}\right| \approx\left|S_{31}\right|
\end{aligned}
$$

On the other hand, accuracy of the coupler termination is critical for the isolation and output reflection coefficients, since $\left|F_{d}\right| \approx 1 / \sqrt{2}$ and $\left|F_{c}\right| \approx 1 / \sqrt{2}$ and we have

$$
\begin{aligned}
\left|\bar{S}_{22}\right| & \approx\left|\Gamma_{0}+F_{d}^{2} \Gamma_{L}\right| \\
\left|\bar{S}_{33}\right| & \approx\left|\Gamma_{0}+F_{c}^{2} \Gamma_{L}\right| \\
\left|\bar{S}_{23}\right| & \approx\left|I_{0}+F_{c} F_{d} \Gamma_{L}\right|
\end{aligned}
$$

When the isolation port of a coupler is terminated with a nonideal shunt resistor with a normalized impedance of $Z_{R}=$ $\rho+j X_{R}$, where $X_{R}$ is the normalized parasitic reactance, the load reflection coefficient $\Gamma_{L}^{a}$ is given by

$$
\left|\Gamma_{L}^{a}\right|=\left|\frac{Z_{R}-1}{Z_{R}+1}\right| .
$$

\section{Termination Network Design for Quadrature Couplers}

We use an accurate and process-tolerant resistive load [10] at the isolation port of a quadrature coupler to cancel out the resistor parasitic effects and increase the tolerance to sheet

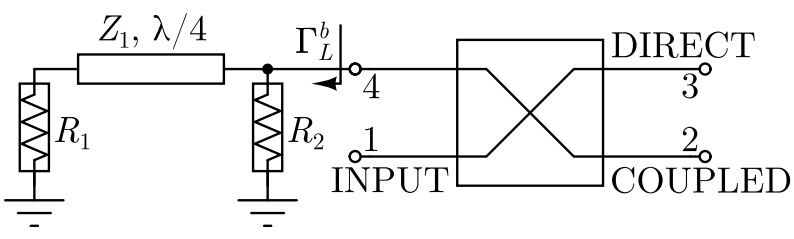

Fig. 2. Proposed termination network at the isolation port of a coupler.

resistance variance. In this way, isolation and output return losses are improved allowing a higher operating frequency.

Our proposed quadrature coupler shown in Fig. 2 is terminated by two shunt resistors separated by a quarter-wave length line. When the resistors are placed closely in the circuit layout, they have the same parasitic effects and normalized sheet resistances. The quarter-wave length line inverts the impedance of the second resistor so that the nonideal effects of the resistors cancel each other. We assume that the deviations in the substrate parameters and the physical line dimensions are negligible [11]. Nevertheless, circuit simulations are repeated with reasonable asymmetry between the resistors and variations in the line impedances, and their effect on our termination network response is limited. Therefore, we rule out such secondary effects and focus on the variations in resistors.

Although resistor values and line impedance can be optimized for the bandwidth and tolerance requirements, we set the resistor values to $2 Z_{R}$ and the line impedance to $2 Z_{0}$, for simplicity. Then, the load reflection coefficient $\Gamma_{L}^{b}$ becomes

$$
\left|\Gamma_{L}^{b}\right|=\left|\frac{T\left(2 Z_{R}-1\right)-T Z_{R}^{2}-j 2\left(Z_{R}^{2}-Z_{R}\right)}{T\left(2 Z_{R}+1\right)+T Z_{R}^{2}-j 2\left(Z_{R}^{2}+Z_{R}\right)}\right|
$$

where $T=\tan (\pi \hat{f} / 2)$ with $\hat{f}=f / f_{0}$. At the center frequency $f_{0}$, when $T \rightarrow \infty$, the proposed termination network achieves twice the return loss (in dB-scale) of a single resistor as

$$
\lim _{T \rightarrow \infty}\left|\frac{\Gamma_{L}^{b}}{\Gamma_{L}^{a}}\right|=\lim _{T \rightarrow \infty}\left|\frac{T\left(Z_{R}-1\right)+j 2 Z_{R}}{T\left(Z_{R}+1\right)+j 2 Z_{R}}\right|=\left|\Gamma_{L}^{a}\right|
$$

regardless of the parasitic effects and the fabrication variances. For a coupler with perfect matching $\left|\Gamma_{0}\right|=0$, any improvement in the termination return loss directly translates as an improvement in the coupler isolation and output return losses as implied by equations (6)-(8).

Usually, quadrature couplers are formed using coupled lines [12] or branch-line coupler based structures [13]. The Lange coupler [14] is also a type of quadrature coupler which employs a multiplicity of interdigitated lines that are connected using crossovers to achieve tight coupling in a wide band. Since Lange couplers have compact size and their crossovers can be easily implement in our MMICs, we demonstrate our termination network with a Lange coupler design.

Our Lange coupler design attempt at $K a$-band, where $X_{R}$ can be as high as 0.4, using the conventional single resistor termination has an output return loss of only $16 \mathrm{~dB}$ even at the center frequency. However, with the proposed termination, the coupler provides output return losses better than $25 \mathrm{~dB}$ with higher tolerance to variations in the process and temperature. 


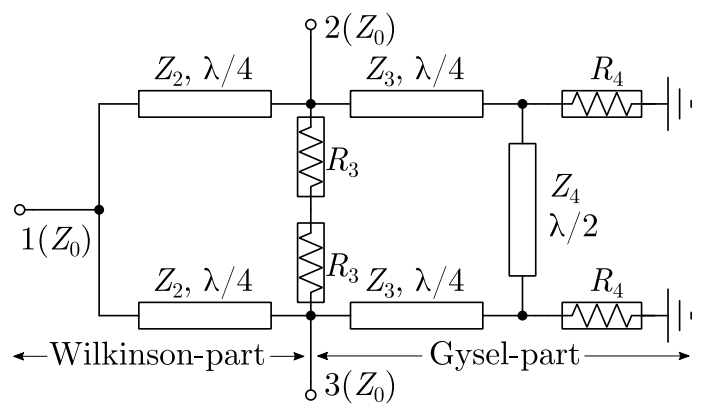

Fig. 3. Divider schematic with the proposed isolation network.

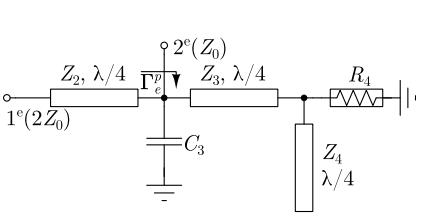

(a)

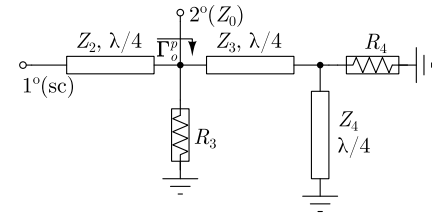

(b)
Fig. 4. Divider (a) even-mode and (b) odd-mode equivalent circuits.

\section{Isolation Network Design for Power Dividers}

The Wilkinson power divider [15] and the Gysel power divider [16] are the two most preferred power dividing structures. Both dividers are easily realizable on a planar circuit and ideally provide low insertion loss, matched conditions at all ports, and high isolation between the output ports. Although the Wilkinson power divider has more bandwidth and occupies less area, the Gysel power divider provides higher power handling capability. However, both dividers suffer from resistor parasitic effects and sheet resistance variance.

To obtain the accuracy and process-tolerance as in the quadrature coupler, we combine the isolation networks of the Wilkinson and Gysel power dividers as shown in Fig. 3. Guan et al. [17] analyzed this structure for increasing the bandwidth and power handling capability. However, a design strategy to cancel resistor parasitic effects and increase tolerance to sheet resistance variance has not been demonstrated before.

We use even-odd mode analysis [18] to calculate the evenmode reflection coefficient $\Gamma_{e}^{p}$ and the odd-mode reflection coefficient $\Gamma_{o}^{p}$ shown in Fig. 4.

In the even-mode, Gysel-part of the circuit becomes negligible in a wide band, because the isolation resistor $R_{4}$ is shorted by $Z_{4}$, and the short circuit is transformed into an open circuit by $Z_{3}$. With no resistor parasitic effects, the Wilkinson divider, the Gysel divider, and the proposed divider simplify to quite similar circuits, and require $Z_{2}=\sqrt{2} Z_{0}$ for perfect matching. As the parasitic capacitance $C_{3}$ of $R_{3}$ increases, the electrical length of $Z_{2}$ can be slightly increased to counterbalance its effect. Also, since the resistors are shorted in a wide band, the even-mode circuits are safe against variations in $\rho$.

In the odd-mode, $Z_{3}$ separates the two impedances $j Z_{2} T / / R_{3}$ and $j Z_{4} T / / R_{4}$ by a quarter-wave length. The latter impedance is inverted and the resulting reciprocal impedance is in parallel with the former impedance. Hence, parasitic effect cancellation and sheet resistance tolerance can be achieved similar to the proposed termination network for the coupler. In the ideal case, for perfect matching we should have

$$
Z_{3}=\sqrt{\frac{R_{3} R_{4} Z_{0}}{R_{3}-Z_{0}}}
$$

and for simplicity we choose $R_{3}=R_{4}=2 Z_{0}$ resulting in $Z_{3}=2 Z_{0}$. This way, the resistors can be sized identically in the circuit layout which makes the resistor parasitic effects similar and is quite helpful if the resistor model is unknown. We note that with known parasitic effects, the values of $Z_{3}, R_{3}$, and $R_{4}$ can be optimized to achieve more bandwidth or a higher sheet resistance tolerance. Although $Z_{4}$ is insignificant for the response at the center frequency and can also be optimized to obtain more bandwidth, we select $Z_{4}=Z_{2}=\sqrt{2} Z_{0}$. Thus, impedances seen from the two ends of $Z_{3}$ are equalized and a better parasitic effect cancellation can be achieved by the symmetrical circuit.

The odd-mode impedance $Z_{o}^{W}$ of a Wilkinson divider is $\sqrt{2} j T / /(\rho / 2+X / 2)$, since we have a two times smaller resistor with a value of $\rho / 2$ and we assume the associated normalized parasitic reactance is also half with a value of $X / 2$. Therefore, at the center frequency, the odd-mode reflection coefficient is

$$
\lim _{T \rightarrow \infty}\left|\Gamma_{o}^{W}\right|=\lim _{T \rightarrow \infty}\left|\frac{Z_{o}^{W}-1}{Z_{o}^{W}+1}\right|=\left|\frac{\rho+X-2}{\rho+X+2}\right|
$$

which is the same for a Gysel divider as $T \rightarrow \infty$. On the other hand, in our proposed divider, the odd-mode impedance $Z_{o}^{p}$ becomes

$$
Z_{o}^{p}=Z_{T} / / 2 \frac{Z_{T}+j 2 T}{2+j Z_{T} T}
$$

where $Z_{T}=\sqrt{2} j T / /(\rho+X)$. At the center frequency, oddmode reflection coefficient of the proposed divider is

$$
\lim _{T \rightarrow \infty}\left|\Gamma_{o}^{p}\right|=\lim _{T \rightarrow \infty}\left|\frac{Z_{T} / /\left(4 / Z_{T}\right)-1}{Z_{T} / /\left(4 / Z_{T}\right)+1}\right|=\lim _{T \rightarrow \infty}\left|\Gamma_{o}^{W}\right|^{2}
$$

and hence twice the odd-mode reflection coefficient (in $\mathrm{dB}$ scale) of a Wilkinson divider is achieved. An improvement in $\Gamma_{o}$ significantly improves the output reflection and isolation coefficients of the divider since they are equal to $\left|\Gamma_{o} / 2+\Gamma_{e} / 2\right|$ and $\left|\Gamma_{o} / 2-\Gamma_{e} / 2\right|$, respectively, and $\Gamma_{e}$ is small as it is a weak function of the resistor nonidealities.

Fig. 5a shows the odd-mode reflection coefficients $\Gamma_{o}^{p}$ and $\Gamma_{o}^{W}$ at the design center frequency $f_{0}$. For the Wilkinson and Gysel power dividers, $20 \mathrm{~dB}$ return loss is barely achieved when there is no variation in $\rho$ for an $\left|X_{R}\right|$ of only 0.2 , while the proposed divider has a return loss better than $20 \mathrm{~dB}$ for $\pm 30 \%$ variation in $\rho$ for an $\left|X_{R}\right|$ up to 0.4 . Also, the normalized $20 \mathrm{~dB}$ bandwidth is deteriorated much less compared to the conventional dividers as shown in Fig. 5b.

\section{FABRICATION AND EXPERIMENTAL RESULTS}

\section{A. Fabrication Technology and Experimental Setup}

A Lange coupler with the proposed termination network and the presented power divider are designed at $K a$-band and fabricated using our GaN-based microstrip technology. To test the tolerance to variations in resistor values, two more variants of 

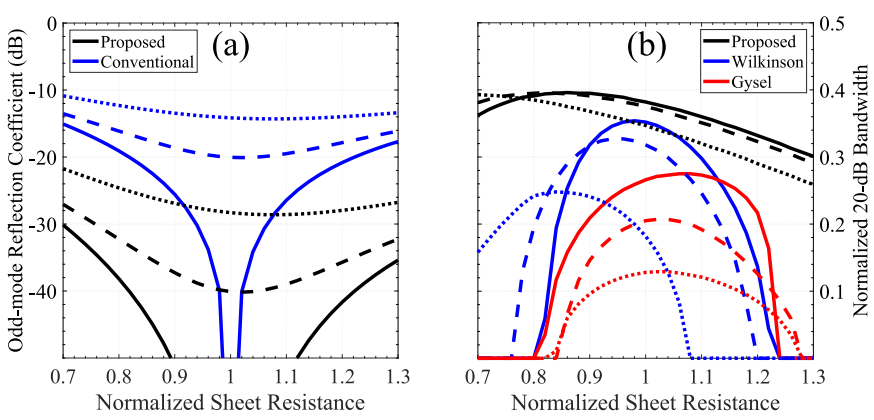

Fig. 5. Calculated: (a) odd-mode reflection coefficients at $f_{0}$ and (b) fractional bandwidths for $X_{R}$ of 0 (solid), \pm 0.2 (dashed), and \pm 0.4 (dotted).

each design are also placed in the same reticle and fabricated in the same run. The additional coupler and divider variants use $30 \%$ smaller and $30 \%$ larger resistors compared to the original designs. Length of the resistors are kept unchanged whereas width of the resistors are varied in the test circuits.

The $100 \mu \mathrm{m}$-thick SiC substrate has a dielectric constant of 9.7. The process uses gold for metalization and TaN sputtering to form thin-film resistors. The topmost metal is $4 \mu \mathrm{m}$ thick. Resistive elements are $90 \mathrm{~nm}$ thick with a sheet resistance of $30 \Omega / \square$. For reference, quarter-wave length at $30 \mathrm{GHz}$ is approximately $1.1 \mathrm{~mm}$ with this technology.

Three-port measurements are performed using Cascade $^{1} \mathrm{RF}$ wafer probe station, $\mathrm{GGB}^{2}$ GSG150 picoprobes and $\mathrm{R} \& \mathrm{~S}^{3}$ ZVA40 vector network analyzer. The measurement results are in good agreement with our simulations, but slightly worse due to the actual process variations in addition to the deliberately modified resistor values.

\section{B. Experimental Results}

Width of the coupler fingers is $14 \mu \mathrm{m}$ with $8 \mu \mathrm{m}$ spacing between them. The termination network uses two $30 \mu \mathrm{m}$ wide high power shunt resistors of $75 \Omega$ and $90 \Omega$ separated by a meandered quarter-wave length transmission line with a characteristic impedance of $90 \Omega$. The resistors share the $85 \mu \mathrm{m}$ wide vias with the measurement pads. Size of the coupler does not increase with the modified termination network and is less than $0.8 \mathrm{~mm} \times 1.55 \mathrm{~mm}$ including the $50 \Omega$ feed lines and on-wafer measurement pads. Microscope photographs of the fabricated Lange couplers are shown in Fig. 6.

Fig. 7 shows the measured S-parameters of the fabricated couplers. Input reflection and transmission coefficients are similar in all variants as expected from equations (3)-(5). The nominal coupler achieves $20 \mathrm{~dB}$ input and output return losses and isolation from DC up to the maximum measurement frequency of $40 \mathrm{GHz}$. The coupled and direct powers are equal at $30.4 \mathrm{GHz}$ with an extra insertion loss less than $0.45 \mathrm{~dB}$, where the phase difference between the output ports is $91.7^{\circ}$.

The measured return loss and isolation levels in all coupler variants are better than $17 \mathrm{~dB}$ from $10.8 \mathrm{GHz}$ to $40 \mathrm{GHz}$

\footnotetext{
${ }^{1}$ Cascade Microtech, Inc., Beaverton, OR, USA.

${ }^{2}$ GGB Industries, Inc., Naples, FL, USA.

${ }^{3}$ Rohde \& Schwarz GmbH \& Company KG, Munich, Germany.
}

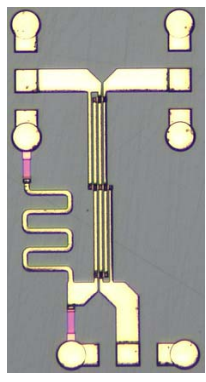

(a)

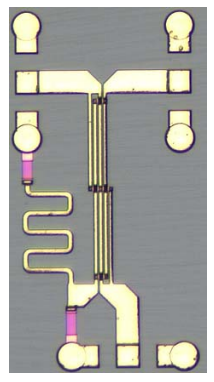

(b)

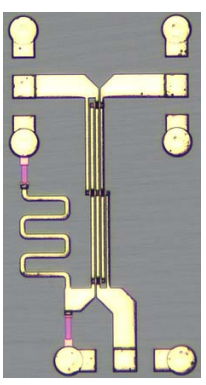

(c)
Fig. 6. Microscope photograph of the couplers with (a) nominal resistors $(\rho=1)$, (b) smaller resistors $(\rho=0.7)$, and (c) larger resistors $(\rho=1.3)$.
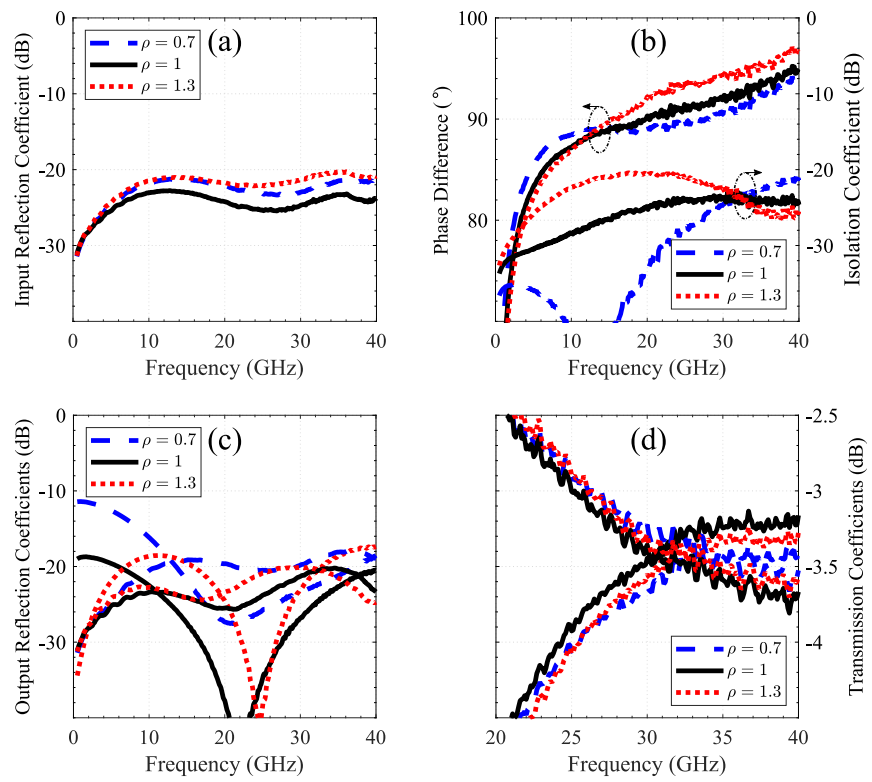

Fig. 7. Measured: (a) $\left|S_{11}\right|$, (b) $\angle S_{21}-\angle S_{31}$ and $\left|S_{23}\right|$, (c) $\left|S_{22}\right|$ and $\left|S_{33}\right|$, and (d) $\left|S_{21}\right|$ and $\left|S_{31}\right|$ of the proposed coupler as a function of frequency.

despite the $30 \%$ change in resistor values. The phase imbalances are within $\pm 3^{\circ}$ from $16.9 \mathrm{GHz}$ to $40 \mathrm{GHz}$. The amplitude imbalances are less than $2 \mathrm{~dB}$ from $22 \mathrm{GHz}$ to $40 \mathrm{GHz}$.

Widths of the quarter-wave length divider lines are $39 \mu \mathrm{m}$, $10 \mu \mathrm{m}$, and $55 \mu \mathrm{m}$ with characteristic impedances of $71 \Omega$, $102 \Omega$, and $63 \Omega$. All resistors are $28.5 \mu \mathrm{m}$ wide. The bridging resistor is $160 \Omega$ and the shunt resistors are $96 \Omega$. Divider size including the $50 \Omega$ feed lines and on-wafer measurement pads is less than $2.58 \mathrm{~mm} \times 1.02 \mathrm{~mm}$. Microscope photographs of the fabricated power dividers are shown in Fig. 8.

Fig. 9 shows the measured S-parameters of the fabricated dividers. The nominal divider achieves $20 \mathrm{~dB}$ input and output return losses and isolation from $19.7 \mathrm{GHz}$ to $32.7 \mathrm{GHz}$ with a fractional bandwidth of $50 \%$.

When the resistor values are changed by $\pm 30 \%$, the lower frequency increases to $21.7 \mathrm{GHz}$, and a fractional bandwidth of $40 \%$ is maintained, demonstrating the tolerance to sheet resistance variance. Extra insertion losses are less than $0.4 \mathrm{~dB}$ in this band, and less than $0.25 \mathrm{~dB}$ at the center frequency.

A comparison with other quadrature couplers (QC) and power dividers (PD) is provided in Table I. This brief stands 


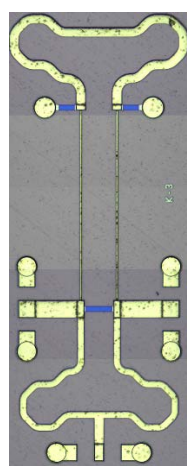

(a)

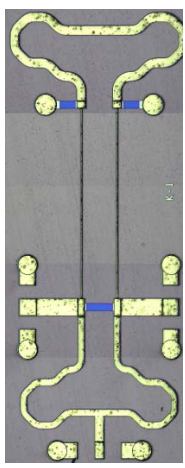

(b)

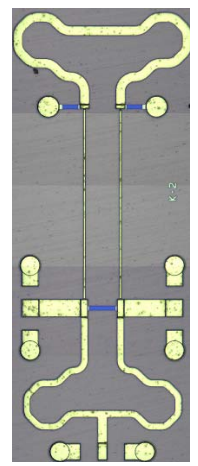

(c)
Fig. 8. Microscope photograph of the dividers with (a) nominal resistors $(\rho=1)$, (b) smaller resistors $(\rho=0.7)$, and (c) larger resistors $(\rho=1.3)$.
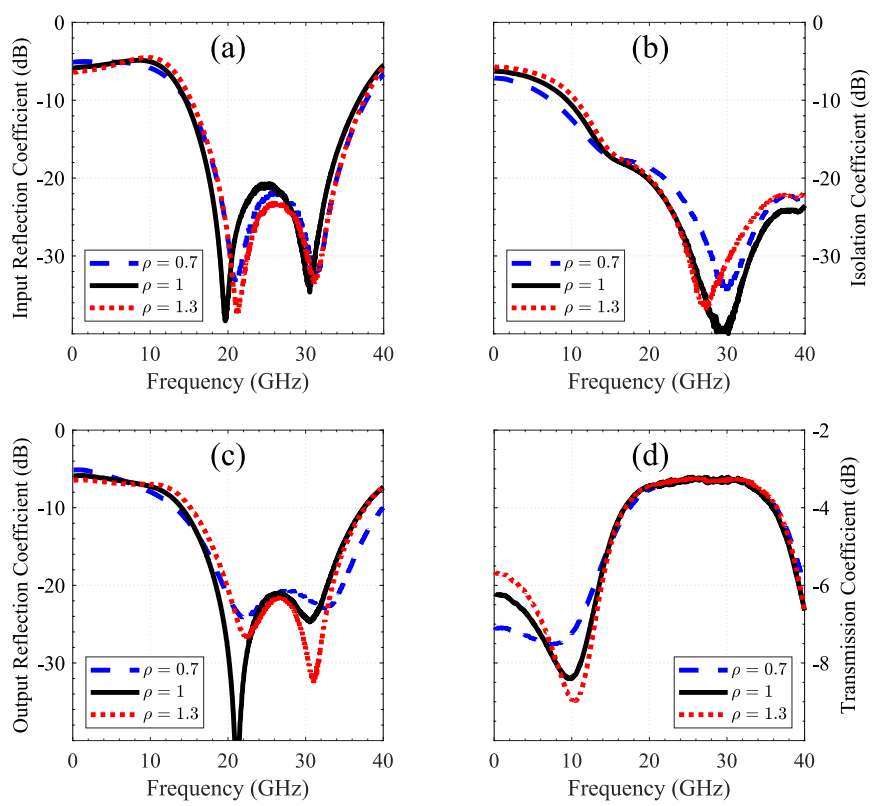

Fig. 9. Measured: (a) $\left|S_{11}\right|$, (b) $\left|S_{23}\right|$, (c) $\left|S_{22}\right|=\left|S_{33}\right|$, and (d) $\left|S_{21}\right|=\left|S_{31}\right|$ of the proposed divider as a function of frequency.

TABLE I

Performance COMPARISON OF IC COUPLERS AND DIVIDERS

\begin{tabular}{|c|c|c|c|c|c|c|c|c|}
\hline Ref. & Type & $\begin{array}{c}f_{0} \\
(\mathrm{GHz})\end{array}$ & $\begin{array}{c}\text { IRL } \\
(\mathrm{dB})\end{array}$ & $\begin{array}{c}\text { ORL } \\
(\mathrm{dB})\end{array}$ & $\begin{array}{c}\text { ISO } \\
(\mathrm{dB})\end{array}$ & $\begin{array}{c}\text { EIL } \\
(\mathrm{dB})\end{array}$ & $\begin{array}{c}20-\mathrm{dB} \mathrm{BW} \\
(\mathrm{GHz})\end{array}$ & $\begin{array}{c}\text { Tolerant } \\
\text { to } \Delta \rho ?\end{array}$ \\
\hline$[3]$ & $\mathrm{QC}$ & 78 & 15 & 12 & $\mathrm{n} / \mathrm{a}$ & 2 & not achieved & no \\
\hline$[4]$ & $\mathrm{QC}$ & 17 & 24 & 16 & 30 & 1.2 & not achieved & no \\
\hline$[5]$ & $\mathrm{PD}$ & 2.4 & 15 & 15 & 38 & 0.4 & not achieved & no \\
\hline$[19]$ & $\mathrm{PD}$ & 10 & 32 & 32 & 20 & 0.45 & $10-12.8$ & no \\
\hline$[20]$ & $\mathrm{PD}$ & 10 & 29 & 23 & 26 & 0.3 & $8.9-11.9$ & no \\
\hline$[21]$ & $\mathrm{QC}$ & 60 & 24 & 24 & 17 & 2.1 & not achieved & no \\
\hline \multirow{2}{*}{$\begin{array}{c}\text { This } \\
\text { work }\end{array}$} & $\mathrm{QC}$ & 30 & 25 & 21 & 24 & 0.45 & DC $-40+$ & yes \\
\cline { 2 - 9 } & & 30 & 32 & 24 & 40 & 0.25 & $20-33$ & yes \\
\hline
\end{tabular}

out with the only circuits tolerant to variations in resistor values. Furthermore, while other high frequency couplers and dividers suffer from parasitic effects, our circuits achieve $20 \mathrm{~dB}$ performance thanks to the presented cancellation technique.

\section{CONCLUSION}

The effect of nonideal resistors in splitting structures is analyzed. An isolation network technique is presented and used to design a Lange coupler and a power divider at $\mathrm{Ka}$-band. High performance in a wide band is achieved while having a sheet resistance tolerance of $30 \%$. Since the resistor parasitic effects increase with frequency the proposed method is quite useful for millimeter-wave circuits.

\section{REFERENCES}

[1] A. A. Coskun and A. Atalar, "Noise figure of a balanced amplifier," IEEE Trans. Circuits Syst. II, Exp. Briefs, vol. 65, no. 9, pp. 1129-1133, Sep. 2018.

[2] V. Napijalo and B. Kearns, "Multilayer $180^{\circ}$ coupled line hybrid coupler," IEEE Trans. Microw. Theory Techn., vol. 56, no. 11, pp. 2525-2535, Nov. 2008.

[3] K. Chan and R. Ramer, "Low-cost E-band Lange coupler with vialess load," Electron. Lett., vol. 51, no. 11, pp. 839-841, May 2015.

[4] A. Bikiny et al., "Ka-band Lange coupler in multilayer thick-film technology," in IEEE MTT-S Int. Microw. Symp. Dig., Boston, MA, USA, Jun. 2009, pp. 1001-1004.

[5] Y. Li, C. Wang, and N.-Y. Kim, "A high performance compact Wilkinson power divider using GaAs-based optimized integrated passive device fabrication process for LTE application," Solid-State Electron., vol. 103, pp. 147-153, Jan. 2015.

[6] S. Chen, W.-C. Lee, and T.-L. Wu, "A resistor-free $N$-way power divider with simultaneous output matching and isolation," IEEE Microw. Wireless Compon. Lett., vol. 29, no. 8, pp. 523-525, Aug. 2019.

[7] Y. F. Pan, S. Y. Zheng, Y. M. Pan, Y. X. Li, and Y. L. Long, "A frequency tunable quadrature coupler with wide tuning range of center frequency and wide operating bandwidth," IEEE Trans. Circuits Syst. II, Exp. Briefs, vol. 65, no. 7, pp. 864-868, Jul. 2018.

[8] T. Zhang and W. Che, "A compact tunable power divider with wide tuning frequency range and good reconfigurable responses," IEEE Trans. Circuits Syst. II, Exp. Briefs, vol. 63, no. 11, pp. 1054-1058, Nov. 2016.

[9] S. J. Mason, "Feedback theory-some properties of signal flow graphs," Proc. IRE, vol. 41, no. 9, pp. 1144-1156, Sep. 1953.

[10] B. Sutbas, E. Ozbay, and A. Atalar, "Accurate and process-tolerant resistive load," IEEE Trans. Microw. Theory Techn., vol. 68, no. 7, pp. 2495-2500, Jul. 2020.

[11] A. Presser, "Interdigitated microstrip coupler design," IEEE Trans. Microw. Theory Techn., vol. 26, no. 10, pp. 801-805, Oct. 1978.

[12] Z. Liu and R. M. Weikle, "A compact quadrature coupler based on coupled artificial transmission lines," IEEE Microw. Wireless Compon. Lett., vol. 15, no. 12, pp. 889-891, Dec. 2005.

[13] R. W. Vogel, "Analysis and design of lumped- and lumped-distributedelement directional couplers for MIC and MMIC applications," IEEE Trans. Microw. Theory Techn., vol. 40, no. 2, pp. 253-262, Feb. 1992.

[14] J. Lange, "Interdigitated stripline quadrature hybrid," IEEE Trans. Microw. Theory Techn., vol. 17, no. 12, pp. 1150-1151, Dec. 1969.

[15] E. J. Wilkinson, "An N-way hybrid power divider," IRE Trans. Microw. Theory Tech., vol. MTT-8, no. 1, pp. 116-118, Jan. 1960.

[16] U. H. Gysel, "A new N-way power divider/combiner suitable for highpower applications," in IEEE MTT-S Int. Microw. Symp. Dig., Palo Alto, CA, USA, May 1975, pp. 116-118.

[17] J. Guan, L. Zhang, Z. Sun, Y. Leng, and Y. Peng, "Designing power divider by combining Wilkinson and Gysel structure," Electron. Lett., vol. 48, no. 13, pp. 769-770, Jun. 2012.

[18] J. Reed and G. Wheeler, "A method of analysis of symmetrical four-port networks," IRE Trans. Microw. Theory Tech., vol. MTT-4, no. pp. 246-252, Oct. 1956.

[19] I. Ju, M.-K. Cho, I. Song, and J. D. Cressler, "A compact, wideband lumped-element Wilkinson power divider/combiner using symmetric inductors with embedded capacitors," IEEE Microw. Wireless Compon. Lett., vol. 26, no. 8, pp. 595-597, Aug. 2016.

[20] B. Sutbas, E. Ozbay, and A. Atalar, "Compact and wideband CPW Wilkinson power dividers for GaN MMIC applications," in Proc. 48th Eur. Microw. Circuit Conf., Madrid, Spain, Sep. 2018, pp. 628-631.

[21] I. Haroun, J. Wight, C. Plett, A. Fathy, and D.-C. Chang, "Experimental analysis of a $60 \mathrm{GHz}$ compact EC-CPW branch-line coupler for mmwave CMOS radios," IEEE Microw. Wireless Compon. Lett., vol. 20, no. 4, pp. 211-213, Apr. 2010. 